\title{
Quark-nucleon dynamics and deeply virtual Compton scattering
}

\author{
Mikhail Gorchtein ${ }^{* \dagger}$ \\ Center for the Exploration of Energy and Matter, Indiana University, Bloomington, IN 47408 \\ E-mail: mgorshte@indiana.edu
}

\section{Adam P. Szczepaniak}

Physics Department and Center for the Exploration of Energy and Matter, Indiana University, Bloomington, IN 47408

E-mail: aszczepa@indiana.edu

\begin{abstract}
We consider deeply virtual Compton scattering and deep inelastic scattering in presence of Regge exchanges. Recently, we have proposed a model in which the diffractive phenomena that are expected to govern the low- $x_{B}$ DIS are incorporated at the parton nucleon level [1, 2]. Such effective parton-nucleon amplitude gives the correct description of low-x structure functions. Surprisingly, however, we have found that in the case of DVCS it breaks collinear factorization, i.e. Bjorken scaling, while it naturally leads to the Regge-type scaling, as it was in fact predicted by Bjorken and Kogut in [3]. In particular, we discuss the contribution of the Pomeron exchange to DVCS in HERA kinematics. A new fit of the DVCS total cross section data from H1 and ZEUS is proposed.
\end{abstract}

XVIII International Workshop on Deep-Inelastic Scattering and Related Subjects, DIS 2010 April 19-23, 2010

Firenze, Italy

\footnotetext{
* Speaker.

${ }^{\dagger}$ This work was supported in part by the US Department of Energy grant under contract DE-FG0287ER40365 and the US National Science Foundation under grant PHY-0555232.
} 

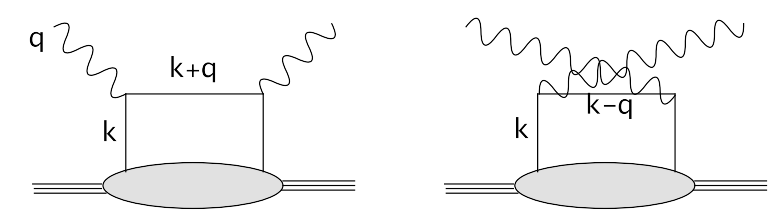

Figure 1: Handbag representation of the Compton amplitude.

In the past two decades, notable theoretical activity has been dedicated to the study of the generalized parton distributions (GPD's) [4]. GPD's allow one to access the nucleon structure in a more detailed manner than the parton distribution functions (PDF's) studied within DIS paradigm, and are a direct generalization of the latter. To access GPD's, it was proposed to study hard exclusive processes, i.e. deeply virtual Compton scattering, (DVCS) $e+p \rightarrow e+p+\gamma$ at high virtuality $Q^{2}$ of the photon originating from the scattered lepton, and low momentum transfer $t$ between recoiled and target nucleon. At present, DVCS has been studied experimentally at HERA [5, 6, 7, 8, 9] and Jefferson Lab [10, 11]. For description of cross section data on DVCS at low-x, two competing formalisms are used, Regge models [12], and the GPD-based models [13]. To be applied phenomenologically, these latter would include models for Regge-like background. In general, Regge background thus represents a systematic effect on the extraction of GPD's. Moreover, if data allow for interpretation without GPD's, one may question the physical content of all GPD models. We focus on applicability of the model $[1,2,14]$ to DVCS in the HERA kinematics, $Q^{2} / W^{2}<<1$.

\section{Compton amplitude, handbag and quark-nucleon amplitudes}

The hadronic Compton tensor is given by the matrix element of the time-ordered product of two electromagnetic currents, $T^{\mu v}=i \int d^{4} z e^{i \frac{q+q^{\prime}}{2}} z\left\langle N\left|T\left[J^{v}(z / 2) J^{\mu}(-z / 2)\right]\right| N\right\rangle$ where $q\left(q^{\prime}\right)$ is the four momentum of the incoming (outgoing) photon. We will consider both the DIS process that corresponds to the forward virtual Compton scattering with both photons spacelike, $q=q^{\prime}$, $q^{2}=q^{\prime 2} \equiv-Q^{2}<0$, and DVCS with $q^{2}<0, q^{\prime 2}=0$ and $\Delta=q-q^{\prime} \neq 0$. The currents are given by $J^{\mu}(z)=\sum_{q} e_{q} J_{q}^{\mu}(z), J_{q}^{\mu}(z)=\bar{\psi}_{q}(z) \gamma^{\mu} \psi_{q}(z)$ with $\psi_{q}$ the quark field operator and $e_{q}$ the quark charge. Using the leading order operator product expansion we replace the product of the two currents by the product of two quark field operators and a free quark propagator between the photon interaction points $z / 2$ and $-z / 2$, as in Fig. 1. In this (handbag) approximation the hadronic Compton amplitude is then given by a convolution $T^{\mu v}=i \int d^{4} K t_{\alpha \beta}^{\mu v}(K, q, \delta) A_{\alpha \beta}\left(K, \Delta, p, \lambda, \lambda^{\prime}\right)$ of the quark Compton tensor

$$
t_{\alpha \beta}^{\mu v}(K, q, \delta)=-e_{q}^{2}\left[\frac{\gamma^{v}\left(\not K+\frac{\not 1+\not q^{\prime}}{2}\right) \gamma^{\mu}}{\left(K+\frac{q+q^{\prime}}{2}\right)^{2}+i \varepsilon}+\frac{\gamma^{\mu}\left(\not K-\frac{q+q^{\prime}}{2}\right) \gamma^{v}}{\left(K-\frac{q+q^{\prime}}{2}\right)^{2}+i \varepsilon}\right]_{\alpha \beta},
$$

$\alpha, \beta$ being Dirac indices, and the untruncated, with respect to the parton legs, parton-nucleon amplitude, $A_{\alpha \beta}\left(K, \Delta, p, \lambda, \lambda^{\prime}\right)=-i \int d^{4} z e^{-i K z}\left\langle p^{\prime} \lambda^{\prime}\right| T\left[\bar{\psi}_{\alpha}(z / 2) \psi_{\beta}(-z / 2)|p \lambda\rangle\right.$. Following [15, 2], we represent this amplitude as

$$
A_{\alpha \beta}\left(K, \Delta, p, \lambda, \lambda^{\prime}\right)=\int d \mu^{2} \sum_{i} I_{n} \frac{\left[\left(k^{\prime}+\mu\right) \Gamma_{i}^{q}(\not k+\mu)\right]_{\alpha \beta}}{\left(k^{\prime 2}-\mu^{2}+i \varepsilon\right)\left(k^{2}-\mu^{2}+i \varepsilon\right)} \bar{u}\left(p^{\prime}\right) \Gamma_{i}^{N} u(p)
$$



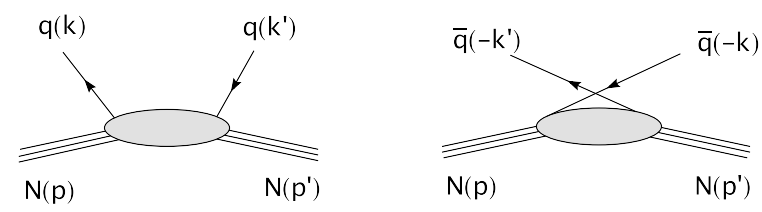

Figure 2: Direct and crossed contributions to the quark - nucleon-scattering amplitude

where $\Gamma_{i, j}^{q, N}$ are constructed from Dirac $\gamma$-matrices and the available four-vectors $p, \Delta, k$. A generic operator $I_{n}=\left(\mu^{2}\right)^{n}\left(d / d \mu^{2}\right)^{n}$ is applied to reflect that the quarks are bound in the nucleon [15].

We proceed by considering the scattering process $N(p)+q(-k) \rightarrow N\left(p^{\prime}\right)+q\left(-k^{\prime}\right)$ shown in Fig.2. Six relevant Dirac-Lorentz structures that can appear in four fermion operators and conserve $P, C P$ and $C P T$, as well as the quark helicity, are listed in Ref. [14] . Here, we concentrate on the Pomeron at $Q^{2} / W^{2} \ll 1$ that corresponds to HERA kinematics. Pomeron contributes to only one structure, $A_{q N}^{\text {Pomeron }}=a_{1} \bar{q} \gamma^{\alpha} q \bar{N} \gamma_{\alpha} N$. The amplitude $a_{1}$ is analytic function of invariants $\hat{s}=(p-k)^{2}=(P-K)^{2}, \hat{u}=\left(p^{\prime}+k^{\prime}\right)^{2}=(P+K)^{2}$ and $t=\Delta^{2}$, has unitarity cuts in $\hat{s}$ and $\hat{u}$ and at fixed- $t$ can be represented through a dispersion representation, $a_{1}\left(\hat{s}, \hat{u}, t, \mu^{2}\right)=$ $(2 \pi)^{4} \int d s\left[\frac{\rho_{1}^{s}\left(s, t, \mu^{2}\right)}{s-\hat{s}-i \varepsilon}+\frac{\rho_{1}^{u}\left(s, t, \mu^{2}\right)}{s-\hat{u}-i \varepsilon}\right]$ with the spectral function $\rho_{1}^{s, u}$ being non-zero above some threshold values $s_{0}\left(u_{0}\right)$ in the respective channel. For fixed $t$ and large $\hat{s}$, we assume the quark-nucleon amplitudes to follow Regge asymptotics, and we find for the spectral functions $\rho_{1}^{s, u} \sim \hat{s}^{\alpha_{P}(t)-1}$, $\alpha_{P}(t)$ being the Pomeron trajectory. An additional constraint on the behavior of the spectral functions comes from the Pomeranchuk theorem which implies that asymptotically $s$ and $u$ channel amplitudes are related $\rho_{1}^{u}(s \rightarrow \infty)=-\rho_{1}^{s}(s \rightarrow \infty)$. We introduce the $C$-even and $C$-odd combinations $\rho_{1}^{\mp} \equiv\left(\rho_{i}^{s} \mp \rho_{i}^{u}\right) / 2$, and notice that $\rho_{1}^{-}$and $\rho_{1}^{+}$correspond to singlet (valence + sea) and non-singlet (valence) GPD's, respectively. We observe that only singlet combination may grow with $s$ in the high energy regime, whereas the non-singlet ones necessarily vanish at high $s$.

\section{Regge exchange contribution to DIS and DVCS}

Next, we will evaluate the contribution to the hadronic Compton amplitude from quark-nucleon amplitude $a_{1}$. We choose the kinematics as $p^{\mu}=\left(p^{+}, 0,0_{\perp}\right)$ and $q^{\mu}=\left(0, Q^{2} /\left(2 x_{B} p^{+}\right), Q_{\perp}\right)$, with the usual Bjorken variable $x_{B}=Q^{2} / 2 p q$, and obtain

$$
\begin{aligned}
& T_{a_{1}}^{\mu v}=-4 i g_{\perp}^{\mu v} \frac{Q^{2}}{x_{B}} \frac{\bar{u}\left(p^{\prime}\right) \gamma^{+} u(p)}{2 P^{+}} \int d \mu^{2} d s \int d^{4} K I_{n} \frac{k_{\perp}^{2}+\mu^{2}}{\left[(K+\Delta / 2)^{2}-\mu^{2}+i \varepsilon\right]\left[(K-\Delta / 2)^{2}-\mu^{2}+i \varepsilon\right]} \\
& \times \rho_{1}^{-}(s)\left[\frac{1}{s-(P-K)^{2}+i \varepsilon}-\frac{1}{s-(P+K)^{2}+i \varepsilon}\right]\left[\frac{1}{\left(K+\frac{q+q^{\prime}}{2}\right)^{2}+i \varepsilon}-\frac{1}{\left(K-\frac{q+q^{\prime}}{2}\right)^{2}+i \varepsilon}\right] \cdot \quad \text { (2.1) }
\end{aligned}
$$

The fact that the above Compton amplitude depends on the singlet spectral function $\rho_{1}^{-}$only, is independent of the collinear approximation: the positive $C$-parity of the Compton amplitude requires the $C$-even singlet combination $\rho_{1}^{-}$. On the contrary, the form factor, possessing the odd $C$-parity only depends on the $C$-odd non-singlet combination $\rho_{1}^{+}$.

\subsection{DIS $\left(\gamma^{*} p \rightarrow \gamma^{*} p\right)$}

We next evaluate the amplitude of Eq.(2.1) in the forward kinematics $\Delta=0, q^{2}=q^{\prime 2}=-Q^{2}$. We make the collinear approximation in the hard quark propagators, $\frac{1}{(k+q)^{2}+i \varepsilon} \approx \frac{x_{B} / Q^{2}}{k^{+} / p^{+}-x_{B}+i \varepsilon}$ and 
$\frac{1}{(k-q)^{2}+i \varepsilon} \approx \frac{-x_{B} / Q^{2}}{k^{+} / p^{+}+x_{B}-i \varepsilon}$, and obtain the familiar result for DIS,

$$
T^{\mu v}(\Delta=0)=g_{\perp}^{\mu \nu} \frac{1}{2 p^{+}} \bar{u}\left(p^{\prime}\right) \gamma^{+} u(p) \int_{0}^{1} d x \frac{2 x}{x^{2}-x_{B}^{2}+i \varepsilon}[q(x)-\bar{q}(-x)]
$$

We can identify the parton densities with integrals over the spectral function,

$$
x[q(x)-\bar{q}(-x)]=-8 \pi^{2} \Gamma(n)(x-1)^{n+1} \int d \mu^{2} d \xi\left(\mu^{2}\right)^{n} \rho_{1}^{+}\left(\frac{\xi}{x}, 0, \mu^{2}\right) \frac{\xi+(n+1-x) \mu^{2}}{\left(\xi+(1-x) \mu^{2}\right)^{n+1}} .
$$

Using high energy asymptotics $\rho_{1}^{+}(s) \sim s^{\alpha_{P}-1}$, with $\alpha_{P}=1+\varepsilon$ the Pomeron trajectory, we obtain the experimentally observed asymptotics $F_{2}\left(x_{B}\right) \sim x_{B}^{1-\alpha_{P}} \sim x_{B}^{-\varepsilon}$.

\subsection{DVCS $\left(\gamma^{*} p \rightarrow \gamma p\right)$}

Next we evaluate Eq.(2.1) in the DVCS kinematics, $p^{\mu}=\left(p^{+}, 0,0_{\perp}\right), q^{\mu}=\left(0, Q^{2} /\left(2 x_{B} p^{+}\right), Q_{\perp}\right)$, $\Delta^{\mu}=\left(-x_{B} p^{+}, 0,0_{\perp}\right)$. Using the collinear approximation for the hard quark propagator $\frac{1}{(k+q)^{2}+i \varepsilon} \approx$ $\frac{x_{B} / Q^{2}}{k^{+} / p^{+}-x_{B}+i \varepsilon}$, and $\frac{1}{\left(k-q^{\prime}\right)^{2}+i \varepsilon} \approx \frac{x_{B} / Q^{2}}{-k^{+} / p^{+}+i \varepsilon}$. The DVCS amplitude in the collinear approximation is

$$
T_{a_{1}}^{\mu v}=g_{\perp}^{\mu \nu} \frac{1}{2 P^{+}} \bar{u}\left(p^{\prime}\right) \gamma^{+} u(p) \int_{0}^{1} d x\left[\frac{1}{x-x_{B}+i \varepsilon}+\frac{1}{x-i \varepsilon}\right] H^{+}\left(x, x_{B}\right),
$$

with the singlet GPD $H^{+}\left(x, x_{B}\right)=\left(1-x_{B} / 2\right) \int_{0}^{1} d y \int_{0}^{1} d z[q(z)-\bar{q}(-z)] \delta\left(x-z-y x_{B}(1-z)\right)$. However, in presence of Regge asymptotics $[q(z)-\bar{q}(-z)] \sim z^{-\alpha_{P}}$, the real part of the integral in Eq.(2.4) is divergent. In the case of the DIS amplitude, this divergency is cancelled in the sum of the direct and crossed handbag diagram. This does not happen in DVCS: one photon is now real, the cancellation between the direct and crossed diagrams is not exact, and cannot compensate for the rise of the GPD at low $x$. For the non-singlet GPD, the integral over $x$ reduces to $\sim d x x^{1-\alpha_{P}}$ and is convergent. Thus we conclude that the collinear approximation is only adequate for valence GPD's, and it is that part of the full DVCS amplitude that obeys Bjorken scaling.

In order to obtain a finite contribution of the singlet GPD to the DVCS amplitude, it is necessary to abandon the collinear approximation in hard propagators [14]. This introduces a scale $\mu^{2}$ associated with the parton-nculeon spectral function, which is absent in the scale-independent collinear approximation. This scale dependence is of no surprise since Regge behavior does introduce a scale. In the limit $Q^{2} / \mu^{2}>>1$ it can be shown that the leading contribution of the Pomeron to the DVCS amplitude has the form [14] $T_{D V C S} \sim Q^{2 \alpha_{P}-2} / x_{B}^{\alpha_{P}} \sim W^{2 \alpha_{P}} / Q^{2}$. This result was obtained in the limit $Q^{2} \rightarrow \infty$. At finite $Q^{2}$, we replace $1 / Q^{2}$ by a $\sim 1 /\left(1+Q^{2} / Q_{0}^{2}\right)$ with some characteristic scale $Q_{0}^{2}$ that we will determine from a fit. We fit the HERA data using the following parametrization for the cross section

$$
\sigma_{\gamma^{*} p \rightarrow \gamma p}=\sigma_{0}\left[\left(\frac{W}{W_{0}}\right)^{\alpha-1}\left(\frac{1}{1+Q^{2} / Q_{0}^{2}}\right)\right]^{2}
$$

with $W_{0}=20 \mathrm{GeV}$. We perform a combined fit to both H1 [5, 6] and ZEUS [7] data. It gives $\sigma_{0}=28 \pm 4 \mathrm{nb}, Q_{0}=1.51 \pm 0.05 \mathrm{GeV}$ and $\alpha-1=0.43 \pm 0.03$ and is shown in Fig 2.2, with $\chi^{2} /$ d.o.f. $=2.01$. We observe that both data sets are fitted well with the Regge form of Eq.(2.5), 

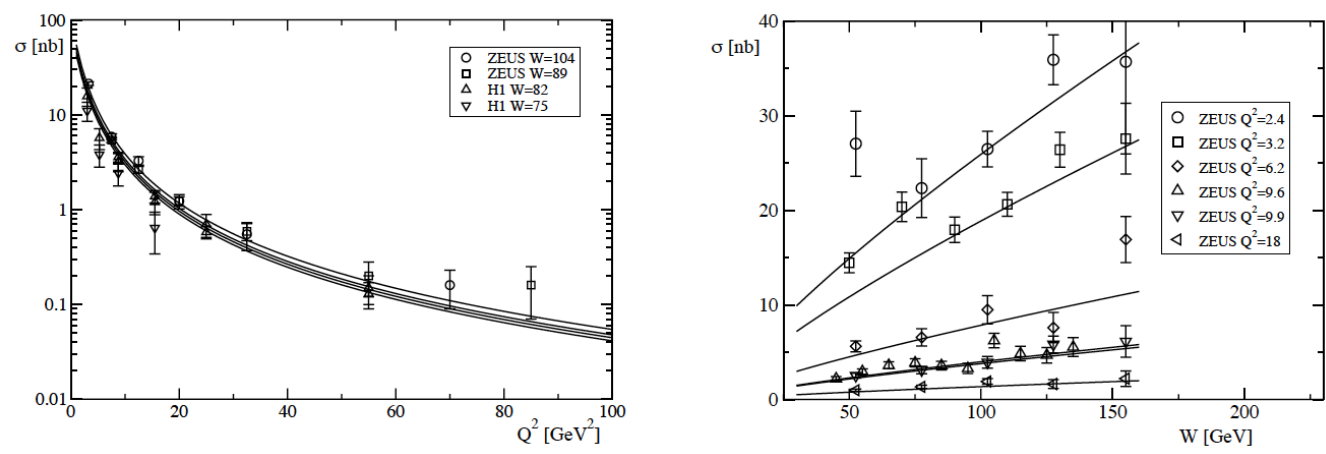

Figure 3: DVCS cross section as function of $Q^{2}$ at fixed $W$ (left panel), and as function of $W$ at fixed $Q^{2}$ (right panel). Solid lines represent a fit to the combined ZEUS and H1 data as explained in the text. Data are from $[5,6,7]$.

as it was found previously in color dipole or Regge based studies [12].

In summary, we presented an analysis of quark-nucleon scattering amplitudes. We considered a basis of six independent Dirac-Lorentz structures and discussed their Regge behavior. Once embedded into the handbag diagram to describe the DVCS amplitude in hard kinematics, we show that only $C$-even (singlet) combinations of the direct and crossed channel contribute. We focused on the contribution of a single Pomeron trajectory that dominates at high energies, and have demonstrate that while for DIS the handbag formalism leads to the known result, $F_{2}\left(x_{B}\right) \sim x_{B}^{-\alpha_{P}}$, in the case of DVCS it leads to divergent integrals in the collinear approximation. If collinear approximation is not used, the model naturally leads to Regge-scaling for DVCS.

\section{References}

[1] A. P. Szczepaniak, T. Londergan, Phys. Lett. B643 (2006) 17.

[2] A. P. Szczepaniak, J. T. Londergan and F. J. Llanes-Estrada, Acta Phys. Polon. B40 (2009) 2193.

[3] J. D. Bjorken and J. B. Kogut, Phys. Rev. D8 (1973) 1341.

[4] X. D. Ji, Phys. Rev. Lett. 78 (1997) 610; A. V. Radyushkin, Phys. Rev. D56 (1997) 5524.

[5] A. Aktas et al., [H1 Collaboration], Eur. Phys. J. C44 (2005) 1.

[6] F. D. Aaron et al. [H1 Collaboration], Phys. Lett. B659 (2008) 796; Phys. Lett. B681 (2009) 391.

[7] S. Chekanov et al. [ZEUS collaboration], Phys. Lett. B573 (2003) 46; JHEP 05 (2009) 108.

[8] A. Airapetian et al. [HERMES Collaboration], Phys. Rev. Lett. 87 (2001) 182001; Phys. Rev. D75 (2007) 011103; JHEP06 (2008) 066; JHEP11 (2009) 083; JHEP06 (2010) 019.

[9] A. Airapetian et al. [HERMES Collaboration], Nucl. Phys. B829 (2010) 1; Phys. Rev. C81 (2010) 035202;

[10] S. Stepanyan et al. [CLAS Collaboration], Phys. Rev. Lett. 87 (2001) 182002.

[11] S. Chen et al. [CLAS Collaboration], Phys. Rev. Lett. 97 (2006) 072002.

[12] A. Donnachie, H. G. Dosch, Phys.Lett. B502 (2001) 74.

[13] V. Guzey, M. V. Polyakov, Eur. Phys. J. C46 (2006) 151.

[14] M. Gorchtein, A.P. Sczcepaniak, Phys. Rev. D in press, [arXiv:1004.2019 hep-ph]

[15] S. J. Brodsky, F. E. Close and J. F. Gunion, Phys. Rev. D8 (1973) 3678. 